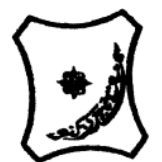

Bayero Journal of Pure and Applied Sciences, 7(1): $62-71$

Received: May 2013

Accepted: March 2014

ISSN 2006 - 6996

\title{
THE EFFECT OF THE SHAPE OF ATOMIC POTENTIAL ON THE DIFFRACTION PATTERN OF ONE DIMENSIONAL QUASICRYSTAL MATERIAL
}

\author{
${ }^{1}$ Sa'id, M., ${ }^{2}$ Babaji, G. and ${ }^{2}$ Gidado, A.S. \\ ${ }^{1}$ Department of Physics, Northwest University, Kano, Nigeria \\ ${ }^{2}$ Department of Physics, Bayero University Kano, Nigeria \\ *Correspondence author: mansursaid79@gmail.com
}

\begin{abstract}
We have simulated the diffraction patterns of both periodic and quasiperiodic monatomic chains using the code Laue (written by Silsbee and Drager) and investigated the effects of the shape of the atomic potential. Three fundamental differences between the diffraction patterns of periodic and quasiperiodic monatomic chain were observed. The width and modulated shape of the diffraction pattern formed by the quasiperiodic chain was found to depend on the shape of the atomic potential. For guassian and exponential atomic shapes, the width decreases as the lattice constant is increased. It also decreases as the size of the atom is increased. For a pseudoatomic shape, the width varies with lattice constant and size of atom in an un-orderly manner.

Keywords: Quasicrystal, Pseudo, Gaussian and Exponential Atomic Potentials
\end{abstract}

\section{INTRODUCTION}

Quasiperiodic crystal, which in a short notation is often called quasicrystal refers to the crystal material with perfect long-range order, but with no three dimensional translational periodicity. Quasicrystals are solid whose diffraction patterns exhibit a rotational symmetry, such as fivefold symmetry that is forbidden for periodic crystal (Fujiwara, et al 2008).

Inspired by the growing number and varieties of quasiperiodic crystal, the international union of crystallography has redefine the term crystal to mean any solid having essentially discrete diffraction diagram, thereby shortening the essential attribute of crystallinity from position space to Fourier space(www.Lassp.cornell.edu/Lifshtz/quasicrystal.html -6). Within the family of crystal, one distinguishes between periodic which are periodic on the atomic scale and aperiodic which are not. This definition reflects our current understanding, that microscopic periodicity is a sufficient but not a necessary condition for crystallinity.

One of the most general features of diffraction pattern displayed by a crystal is that, each Bragg's peak in discrete diffraction pattern determines a wave vector at which the electron density has non vanishing coefficient in its Fourier expansion. In quasicrystal there is existence of the other peaks between the already existing peaks. This is because quasicrystal lattice are dense in the mathematical sense. This implies that, there are no requirements of minimal distance between wave vectors. Hence the absence of such requirement is what allows lattice to have symmetries which are forbidden in periodic crystal ( $n$ fold rotations, with $n=5$ and $>6$ ).

The two forms of quasicrystal are; Icosahedral quasicrystal and Polygonal quasicrystal

- Icosahedral quasicrystal is characterized by the occurrence of sharp diffraction spot.

- Polygonal quasicrystal is characterized by the occurrence of non-crystallographic rotational symmetry.
The history of quasicrystal begins with the 1984 paper (Shechtman et al., 1984) "Metallic phase with longrange orientation order and no translation symmetry" where D. shechtman et al demonstrated a clear diffraction pattern with a five-fold symmetry. The pattern was recorded from an Al-Mn alloy which has been rapidly cooled after melting. Ishimasa et al, (1985), reported twelve fold symmetry in $\mathrm{Ni}-\mathrm{Cr}$ particles. Over the years, hundreds of quasicrystals with various compositions and different symmetries have been discovered. The first quasicrystalline materials were thermodynamically unstable-when heated. The first of many stable quasicrystals(e.g AlCoNi) were discovered making it possible to produce large samples for study and opening thedoor to potential applications(Lifshitz, 2003).

Ferralis et al. (2004) investigatethe diffraction from One- and two dimensional quasicrystalline gratings" using two different approaches (laser diffraction and calculated diffraction method using Fibonacci series). The work suggested that, laser diffraction allows an inductive approach for understanding complex aperiodic structure, and can provide researchers with an innovative tool for introducing and extending the traditional concept of diffraction. In 1985, Levine conducted a theoretical work on the diffraction pattern for an ideal model of quasilattice in which identical atoms are placed at each point of the lattice, and obtained results that are in agreement with the observation earlier reported by Shechtmen et al., (Shechtman, 1984).

To generate a quasicrystal patterns, projection methods have been developed (De-Bruijin, 1981; Kramer et al,. 1984 and Dueau, 1984). Projection methods are mathematical constructs that projects sections of hypercubic lattice onto lower dimensional spaces. The first such projection was given by DeBruijn (Latvin, 1988), where he showed that the vertices of the two-dimensional penrose pattern of darts and kites can be generated by hypercubic lattice into a one-dimensional space. 
In this work, the effects of varying the shape of the atomic potentials are investigated on one-dimensional quasicrystal material using the code 'Laue' written by Silsbee and Drager,(2005). Laue is based on the projection technique, however, unlike traditional projection work, in which the emphasis is entirely on the diffraction pattern, the code can provide additional data that can be used to characterize the quasicrystal. Three types of potentials namely Gaussian, Exponential and Pseudo atom were used in this work. The electron density, $\rho(x)$ at distance $x$ from the atomic center of a single atom for the potentials are given in Table 1 below. In these expressions, $R$ is the atomic position, $\mathrm{Z}$ its atomic number, and $\sigma$ is the size of the atom which is taken as the root mean square width of its electron density. The pseudo atom is represented by an electron density of the core electrons, a Gaussian of width $\sigma_{\sigma}=\sqrt{\frac{2}{17 \sigma}}$, superimposed on an electron density of the valence electrons, a Gaussian of width $\sigma_{w}=\sqrt{\frac{32}{17 \sigma}}$. All three atom shapes agree in their zeroth, first, and second moment (i.e $\int_{-\infty}^{\infty} d x x^{n} \rho(x)$ is the same for each $\rho(x)$ for $n=0,1,2$.

Table 1 Expression for the Electron Density.

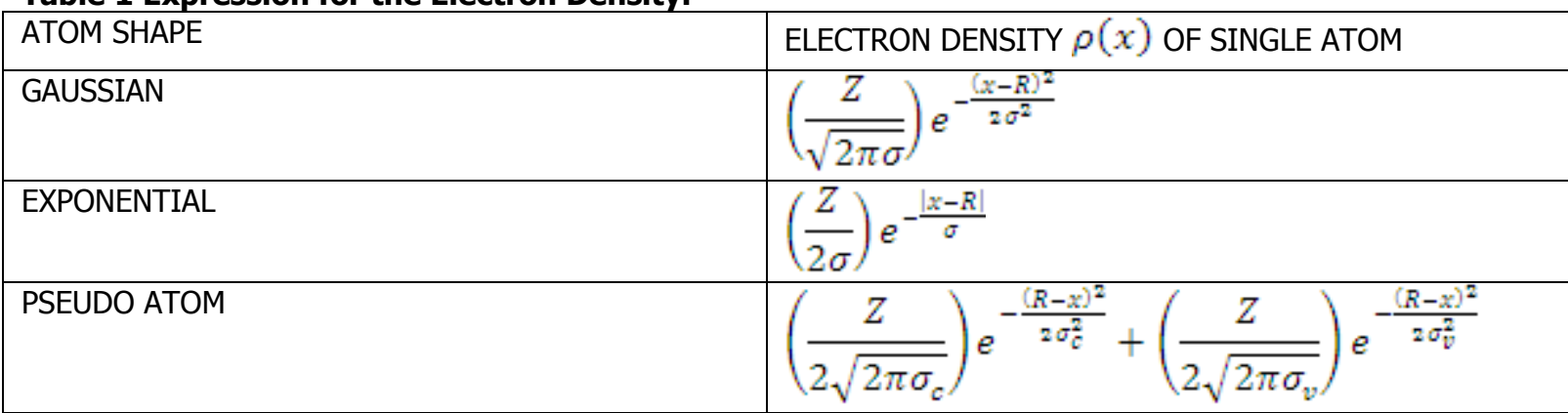

\section{DIFFRACTION PATTERN OF QUASICRYSTAL}

The diffraction patterns of the quasicrystal consist of a set of Bragg peaks that densely fill reciprocal space in an array with quasicrystal symmetry. For a one-Dimensional quasicrystal with atomic position of the $\mathrm{N}^{\text {th }}$ atom given by(Levine 1985);

$$
X_{N}=N+\alpha+\frac{1}{\pi}\left[\frac{N}{\pi}+\beta\right],
$$

where $\tau$ is thegolden ratio; $\alpha$ and $\beta$ are arbitrary real numbers and $\left[\frac{N}{\tau}+\beta\right]$ 's represent the greatest integer function. This particular example is central to study the pentagonal and icosahedral quasilattice. The atomic position of the 1D (Fibonacci) quasicrystal described by Eqn. (1) may be expressed as;

$$
X_{N}=n\left(1+\frac{1}{\pi^{2}}\right)+\beta \sqrt{5}+\left[-\frac{1}{\tau}\left\{\frac{n\left(1+\frac{1}{\pi^{2}}\right)+\beta \sqrt{5}}{\sqrt{5}}\right\}-\beta \tau+\alpha\right] \text {, }
$$

where $\left\{\frac{n\left(1+\frac{1}{\pi^{2}}\right)+\beta \sqrt{5}}{\sqrt{5}}\right\}$ signifies the fractional part function and we have used the fact that $\left(1+\frac{1}{\pi^{2}}\right)=\sqrt{5}$. Using identity is $X=[X]+\{X\}$.The function $\{X\}$ is periodic in $\mathrm{X}$ with period 1 . This expression is of the general form;

$$
X_{n}=n a+\varphi+F(n \alpha+\varphi),
$$

where $F(x)$ is periodic in $x$ with period $b_{y}$ and $\frac{a}{b}$ is irrational. Expressions of this variety arise in the study of Frenkel-Kontrovamodel(Lavine, 1985), which describe a 1D in commensurate crystal. The Fourier transform of such a set of atomic positions consist of Bragg peaks at positions

$$
K=\frac{2 \pi \mathbb{M}}{a}+\frac{2 \pi W}{b}
$$

where $\mathrm{M}$ and $\mathrm{N}$ are integers. This result may be obtained by expanding the exponential $e^{i \mathrm{ikF}(\mathrm{n} a+\varphi)}$ appearing in the expression for the transform in a Fourier series of its own and employing the completeness relation for complex exponentials. For our case, this means that there will be peaks at

$$
K_{\mathrm{pq}} \equiv \frac{2 \pi}{1+\frac{1}{\pi^{2}}}\left(p+\frac{q}{\pi}\right)
$$

where $\mathrm{p}$ and $\mathrm{q}$ are integers. The diffraction pattern (i.e. Fourier transform of the $1 \mathrm{D}$ quasicrystal can be computed using this relation from Eqn. (2). 


$$
F_{i}(K)=\lim _{N \rightarrow \infty} \frac{1}{N} \sum e^{i K x_{N}},
$$

where the summation is over the $\mathrm{N}$ atomic position in the chain. First consider $F_{i}(K)$ for $\mathrm{K}$ of the form $K=K_{\mathrm{pq}}$ as define in eqn. (4). Noting the identitythe exponent in eqn. (5) is given by;

$i K_{\mathrm{pq}} X_{n}=2 \pi i\left(p n+q \frac{n}{\tau}\right)+K_{\mathrm{pq}}\left(\frac{\beta}{\tau}+\alpha-\frac{1}{\tau}\left\{\frac{n}{\tau}+\beta\right\}\right)$
$=2 \pi i\left(p n+q\left[\frac{n}{\tau}+\beta\right]\right)+i\left(2 \pi q-\frac{K_{p q}}{\tau}\right)\left\{\frac{n}{\tau}+\beta\right\}+i K_{p q} \alpha-i\left(2 \pi q-\frac{K_{p q}}{\tau}\right) \beta(6)$

The first term in the expression $2 \pi i\left(p n+q\left[\frac{n}{\tau}+\beta\right]\right)$ is an integer times $2 \pi i$ and therefore only yield a factor of unity upon exponentiation. The last two terms $i K_{\mathrm{pq} q} \alpha-i\left(2 \pi q-\frac{K_{\mathrm{pq} q}}{\tau}\right) \beta$ are independent and contributes to the sum in an important fashion. Since $0 \leq\left\{\frac{n}{\tau}+\beta\right\} \leq 1$ the second term $i\left(2 \pi q-\frac{K_{y q}}{\tau}\right)\left\{\frac{n}{\tau}+\beta\right\}$ lies between zero and $i X$, where $X \equiv 2 \pi q-\frac{K_{y q}}{\tau}$ since $\tau$ is an irrational number, the value of the second term is uniformly and densely distributed in the interval $(0, \mathrm{X})$, enabling us to approximate the sum in eqn. (5) by an integral;

$$
F_{i}(K)=\frac{e^{i \omega}}{X} \int_{0}^{X} e^{i y} d y=\frac{\sin \frac{X}{x}}{\frac{X}{x}} e^{i y},
$$

where $\square \equiv K_{\mathrm{pq}} \alpha-\left(2 \pi q-\frac{K_{\mathrm{yq}}}{\tau}\right) \beta$ and $\gamma \equiv \mathbb{\square}+\frac{z}{2}$. Equation (7) is the solution of $F_{\mathrm{x}}(K)$ for the special values $K=K_{\mathrm{pq}}$, which correspond to the position of bragg peaks. Thus we conclude that

$$
F_{i}(X)=\sum_{p q}\left(\frac{\sin \frac{X}{x}}{\frac{X}{x}} e^{i y}\right) \delta\left(K-K_{p q}\right) .
$$

Those familiar with the computation of the fourier transform of 1D quasicrystal via projection method will recognize that the two methods agree exactly.

The brightest spots occur for those $K=K_{\mathrm{pq}}$ where $X$ is small. This occurs when $\frac{q}{p}$ is close to $\tau$. It is well known that the best rotational approximants to $\tau$ occur when $\mathrm{q}$ and $\mathrm{p}$ are successive Fibonacci number $F_{n}$. This means that the sequence of most intense peaks corresponds to $(p, q)=\left(F_{n+1}, F_{n}\right)$ (Levine, 1985) .

The diffraction pattern of a quasicrystal is one of its most distinctive features, the pattern consist of a set of Bragg's peaks that densely fill reciprocal space. This result can be understood intuitively by considering the case of 1D quasicrystal with atomic position given in equation (1). The equation can be divided into a sum of two functions that describe periodic spacing but with incommensurate periods. If the first term were kept, the diffraction would consist of Bragg peaks spaced periodically in reciprocal space with some fundamental period $K_{0}$. Because the second term is incommensurate, it leads to Bragg peak at some incommensurate reciprocal space period $K_{1}$. The full pattern then consist of the union of the two sets of peak plus peaks at linear combination of $K_{0}$ and $K_{1}$. In this case of simulating quasicrystal, the program has the following variables: Lattice constant, Size of
Because the two are incommensurate, the peaks densely fill reciprocal space (in a countable non-fractal way) (Levine, 1985)

\section{MATERIALS AND METHODS Laue Code}

The code, "Laue" written by Silsbee and Drager (2005) was employed in this work. Basically the code computes the electron density and diffraction pattern of a one-dimensional array of atoms. The onedimensional crystal is represented by its real-space electron density which is generated from superposition of atomic electron densities. The corresponding diffraction is then computed as the square of the Fourier transform of that electron density. To compute the diffraction pattern (Intensity) of the electron density, "Laue" uses a Fast Fourier Transform (FFT) routine. The intensities are normalized to give a height for the central peak of one for the monatomic quasicrystal.

The program has two main output windows for displaying the electron density and diffraction pattern respectively. The code has eight menus namely: Quit Display, Configure, Presets, Help, Material, Modulation and Calculate. The material menu allows user to select the type of material simulated. The possible options are Monatomic crystal, Diatomic crystal, Single atom, Pair of atoms, Liquid and Quasicrystal.

atom, the Spacing ratio and Atom shape. In this work the atomic potential was first chosen to be Gaussian, 
and then followed by Pseudo and Exponential and in each, the diffraction pattern was observed and analyzed.

\section{Procedure \\ Procedure 1(Monatomic Chain)}

The size of the atom was set to be 0.1 Aand the atomic potential was chosen to be Pseudo. The lattice constant was set to be $3.0 \AA$ and then the simulation executed. The procedure was repeated for the values of lattice constant of $4.0,5.0,6.0$ and $7.0 \AA$. The data from the simulation was then exported to excel for further analysis which include among others, computation of the width of the diffraction pattern and plotting the variation of diffraction intensity with the scattering wave vector for the following values of size of the atom; $0.2,0.3,0.4,0.5,0.6$ and $0.7 \AA$.

\section{PROCEDURE 2 (QUASICRYSTALS)}

The atomic potential shape was chosen to be Gaussian, the golden mean, the size of the atom and the lattice constant were set at $1.6,0.1 \AA$ and $3.0 \AA$ respectively. The code was then run. The lattice constant was varied to $4.0,5.0,6.0$ and $7.0 \AA$ respectively and the simulation executed for each of lattice constant.
The size of the atom was then varied to $0.2,0.3,0.4$, $0.5,0.6$ and $0.7 \AA$ respectively and this procedure the was repeated for each atomic size. The data obtained for the diffraction due to Gaussian potential was exported for further analysis.

The atomic potential shape was changed to pseudo and exponential respectively and the procedures above were repeated.

\section{RESULTS AND DISCUSSION}

It is important to understand the units of intensity used in the plots of the diffraction pattern and the width of the diffraction pattern. In the plots of the diffraction pattern for the periodic monatomic chain, the intensities of the peaks are normalized such that the central peak is unity. Thus in these plots the intensities of all the central peaks are unity in arbitrary units even though their actual magnitudes are not equal. In the plots of the diffraction pattern for the quasi periodic monatomic chain, the intensities are not normalized but are also given in arbitrary units.

The width of the diffraction pattern simply refers to the range $\left[-\Delta k_{y} \Delta k\right]$ where the intensity is identically zero. This is not the same as the width of the distribution function. It is also not the same as the width that is visible in the plots of the diffraction pattern.

\section{Features of the Diffraction Pattern of A Periodic Monatomic Chain}

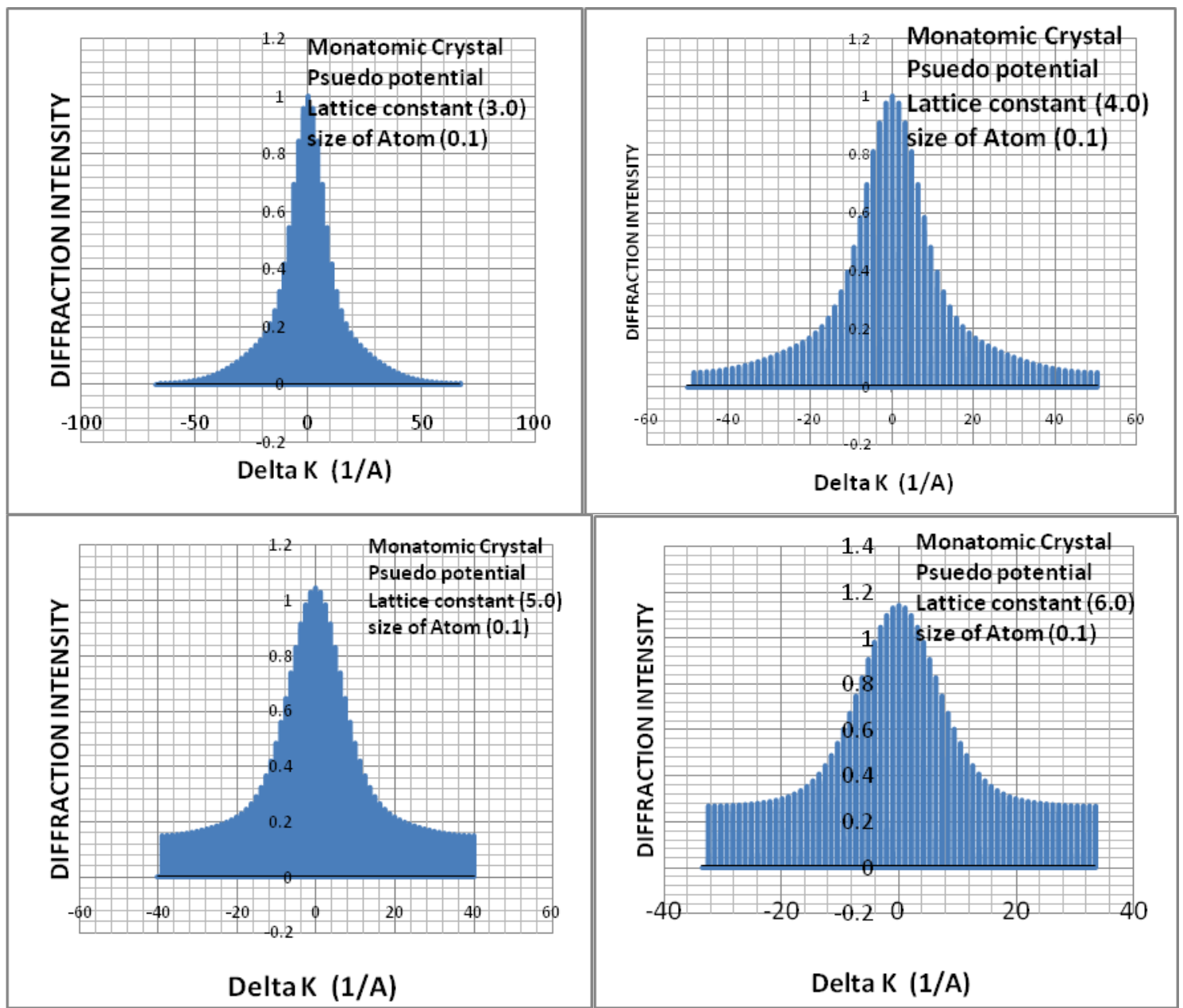




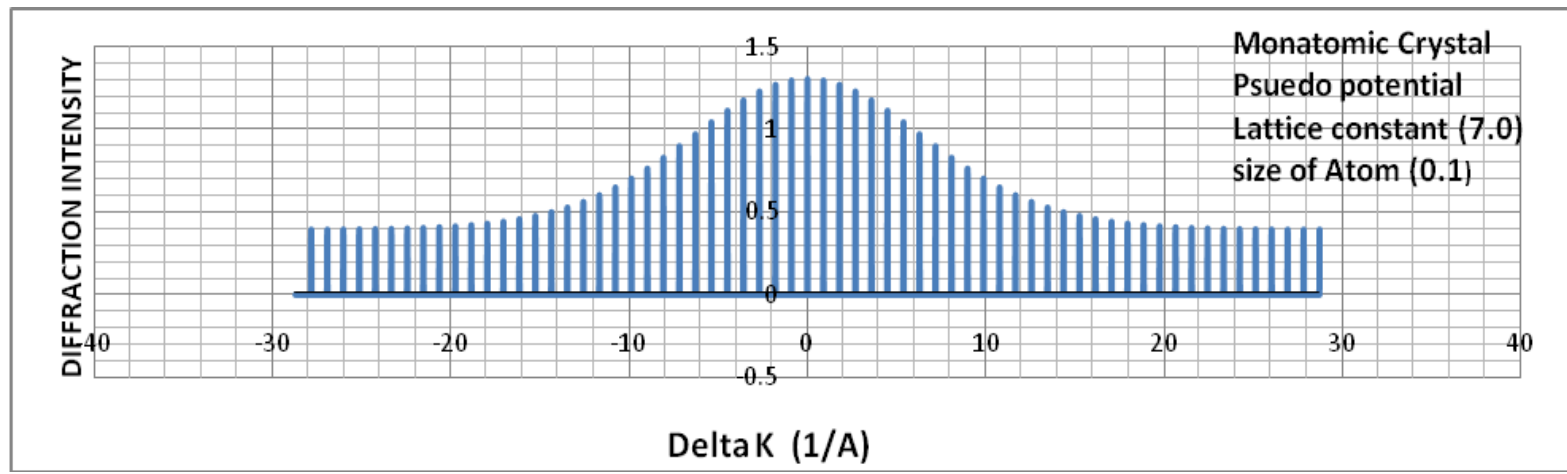

Figure 1: Sample of the Diffraction Pattern for Periodic Monatomic Chain using Pseudo Potential

The following features of the diffraction pattern of the monatomic chain (atomic potential being pseudo) are observed from Fig.1:

i. The diffraction peaks are equally spaced, i.e the scattering wave vector, $\Delta k$ is constant.

ii. The intensity of the peaks is inversely proportional to $|\Delta k|$.

iii. Since each Bragg's peaks determine the wave vector, the Bragg's planes of the material are equally spaced, ensuring the periodicity of the material.

iv. For a given atomic size, as the lattice constant increases, the diffraction intensity for the central peak increases.

v. From Table 1.below, the width of the diffraction pattern varies in an un-oderly manner with respect to size of atom and lattice constant. This will allows the careful observation for the variation of lattice constant with the increase of size of the atom.

Table 1: Variation of width of diffraction patter with size of the atom for Pseudo Potential

\begin{tabular}{|c|l|c|c|c|c|}
\hline $\begin{array}{c}\text { Lattice constant } \\
(A)\end{array}$ & $\mathbf{3 . 0}$ & $\mathbf{4 . 0}$ & $\mathbf{5 . 0}$ & $\mathbf{6 . 0}$ & $\mathbf{7 . 0}$ \\
\hline & & & & & \\
\hline & & & & & \\
\hline 0.1 & & & & & \\
\hline 0.2 & 131.9468 & 98.9602 & 79.1681 & 65.9734 & 56.5486 \\
\hline 0.3 & 119.3805 & 98.9602 & 79.1681 & 65.9734 & 56.9734 \\
\hline 0.4 & 67.0206 & 69.1150 & 70.3716 & 65.9734 & 56.5486 \\
\hline 0.5 & 50.2654 & 50.2654 & 52.7788 & 52.3598 & 52.0606 \\
\hline 0.6 & 131.9468 & 40.8408 & 40.2124 & 41.8880 & 41.2896 \\
\hline 0.7 & 131.9468 & 98.9602 & 35.1858 & 33.5104 & 34.1088 \\
\hline
\end{tabular}

Features of The Diffraction Pattern of The Quasiperiodic Monatomic Chain Guassian Potential

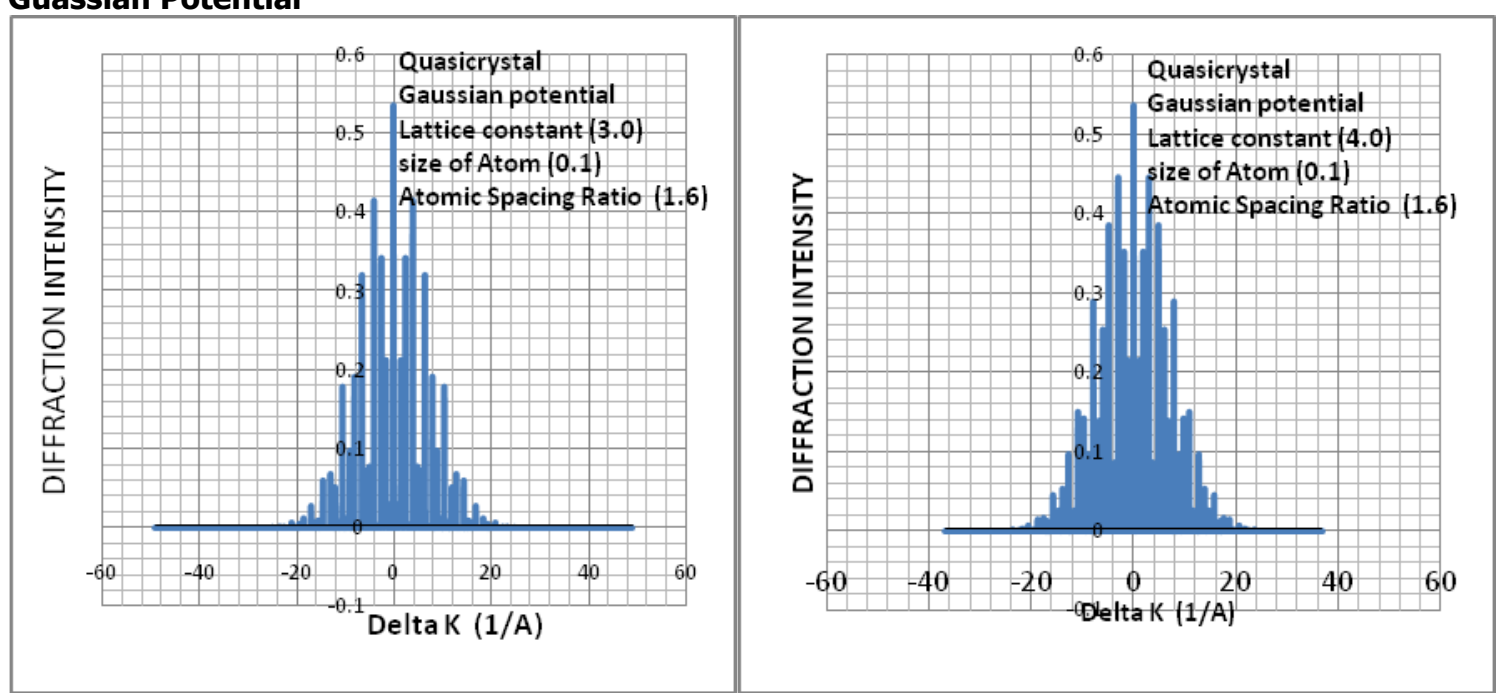




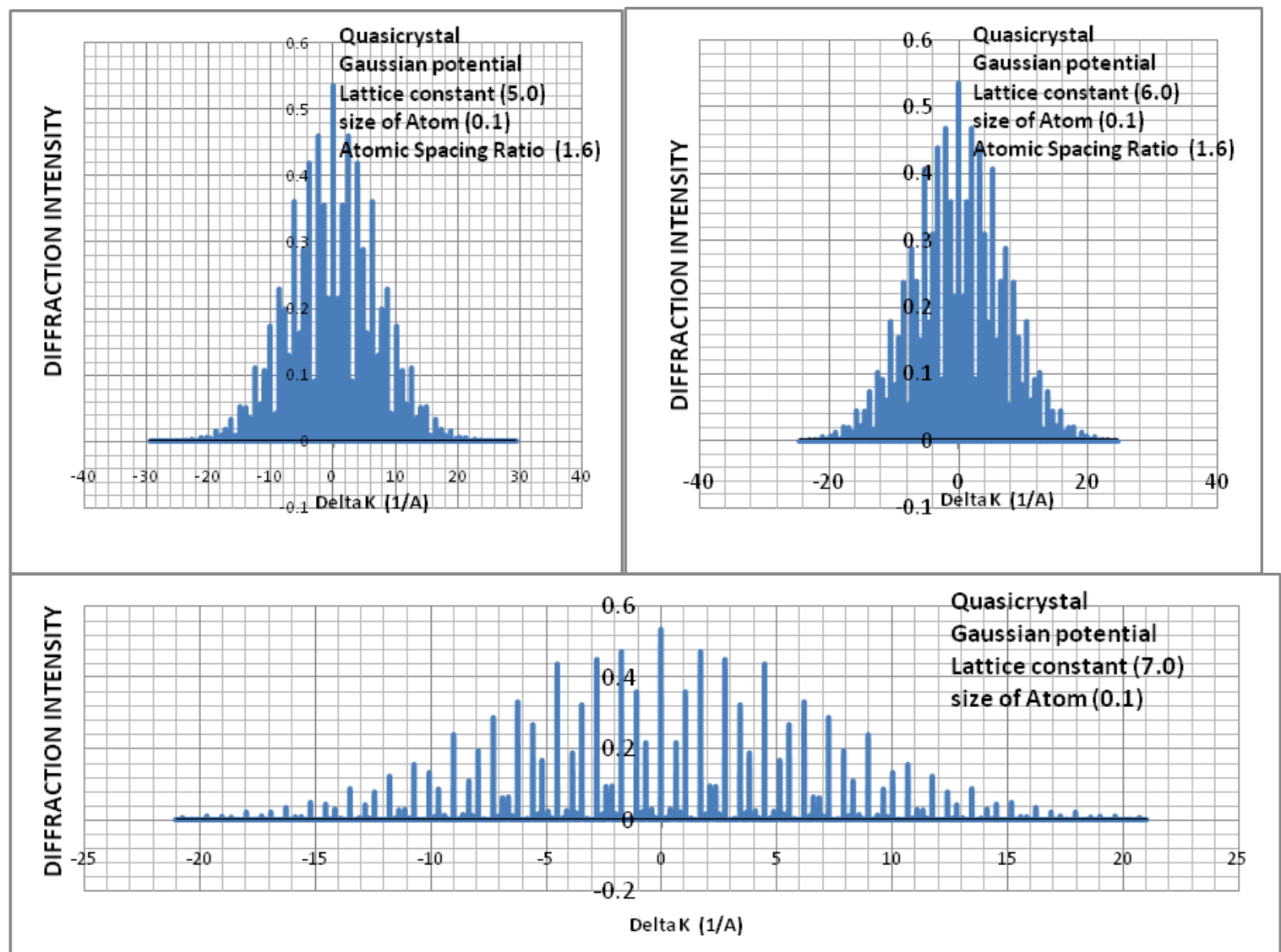

Figure 2: Sample of the Diffraction Pattern for Gaussian Potential

The following features of the diffraction pattern of the Quasicrystal (atomic potential being Gaussian) are observed from Fig.2:

i. Each diffraction pattern has sharp peaks, just like periodic crystal, but in addition there is a background intensity.

ii. The spatial distribution of the diffraction peaks is unique for a given size of atom, in particular the spacing between the peaks is constant for a given size of atom and independent of the lattice constant.

iii. The intensity of the central peak is maximum and in general, the intensity decreases as the scattering wave vector is increased, however, the intensity is not strictly proportional to the scattering wave vector.

iv. For a given size of atom, there is an increase in the background intensity, as the lattice constant increases.

v. For a given lattice constant, the width of the diffraction pattern decreases as the size of the atom increases as shown in Table 2. However, for a given size of atom the width varies in an unorderly manner with respect to lattice constant.

Table 2: Variation of width of diffraction pattern with size of the atom for Gaussian Potential

\begin{tabular}{|c|c|c|c|c|c|}
\hline Size of Atom & $\mathbf{3 . 0}$ & $\mathbf{4 . 0}$ & $\mathbf{5 . 0}$ & $\mathbf{6 . 0}$ & $\mathbf{7 . 0}$ \\
& & & & & \\
& & & & & \\
\hline & & & & & \\
\hline 0.1 & & & & & \\
\hline 0.2 & 30.8356 & 72.5550 & 58.6334 & 48.9811 & 42.0249 \\
\hline 0.3 & 33.8842 & 53.1182 & 35.8588 & 35.4178 & 35.9040 \\
\hline 0.5 & 24.0112 & 23.1126 & 23.2924 & 23.4122 & 23.4978 \\
\hline 0.6 & 17.8766 & 18.0084 & 17.3686 & 16.9421 & 17.9516 \\
\hline 0.7 & 12.9402 & 13.4075 & 14.4068 & 14.4739 & 14.5218 \\
\hline & 11.0712 & 12.0056 & 10.7547 & 12.0056 & 11.0916 \\
\hline & 9.8729 & 9.7051 & 9.6045 & 10.4720 & 10.2905 \\
\hline
\end{tabular}




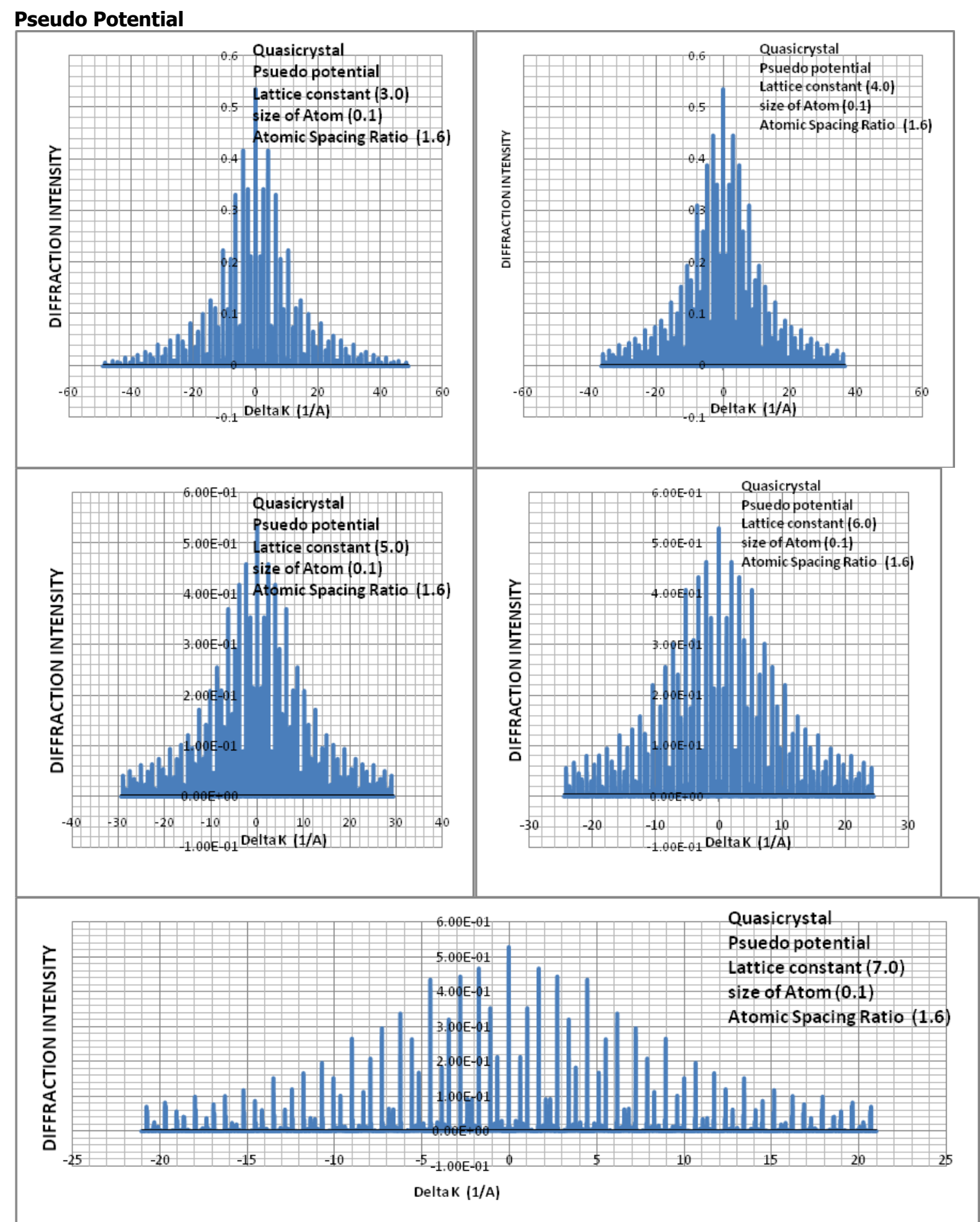

Figure 3: Sample of the Diffraction Patterns for Pseudo Potential

The following features of the diffraction pattern of the Quasicrystal (atomic potential being Pseudo) are observed from Fig. 3:

i. Each diffraction pattern has sharp peaks, just like periodic crystal, but in addition there is a background intensity.

ii. The spatial distribution of the diffraction peaks is unique for a given size of atom, in particular the spacing between the peaks is constant for a given size of atom and independent of the lattice constant. iii. Intensity of the central peak was found to be the same as that of guassian potential.

iv. For each size of the atom there is higher (compared to the guassian potential) increase in the background intensity as the lattice constant increases.

v. The width of the diffraction pattern varies in an un-oderly manner with respect to size of atom and lattice constant as shown in Table 3. 
Table 3: Variation of width of diffraction patter with size of the atom

\begin{tabular}{|c|c|c|c|c|c|}
\hline $\begin{array}{c}\text { Lattice constant } \\
(A)\end{array}$ & $\mathbf{3 . 0}$ & $\mathbf{4 . 0}$ & $\mathbf{5 . 0}$ & $\mathbf{6 . 0}$ & $\mathbf{7 . 0}$ \\
\hline & & & & & \\
\hline & & & & & \\
\hline 0.1 & & & & & \\
\hline 0.2 & 97.8579 & 73.5615 & 58.8779 & 49.0605 & 42.0557 \\
\hline 0.3 & 96.7160 & 73.2918 & 58.7772 & 49.0290 & 42.0351 \\
\hline 0.4 & 96.7160 & 65.8992 & 66.5342 & 58.0296 & 48.8612 \\
\hline 0.5 & 49.8916 & 49.4244 & 50.2654 & $48 . .3580$ & 41.4702 \\
\hline 0.6 & 38.8206 & 37.4188 & 39.5358 & 39.4196 & 39.3340 \\
\hline 0.7 & 32.0150 & 31.4160 & 32.8968 & 32.9496 & 33.7882 \\
\hline
\end{tabular}

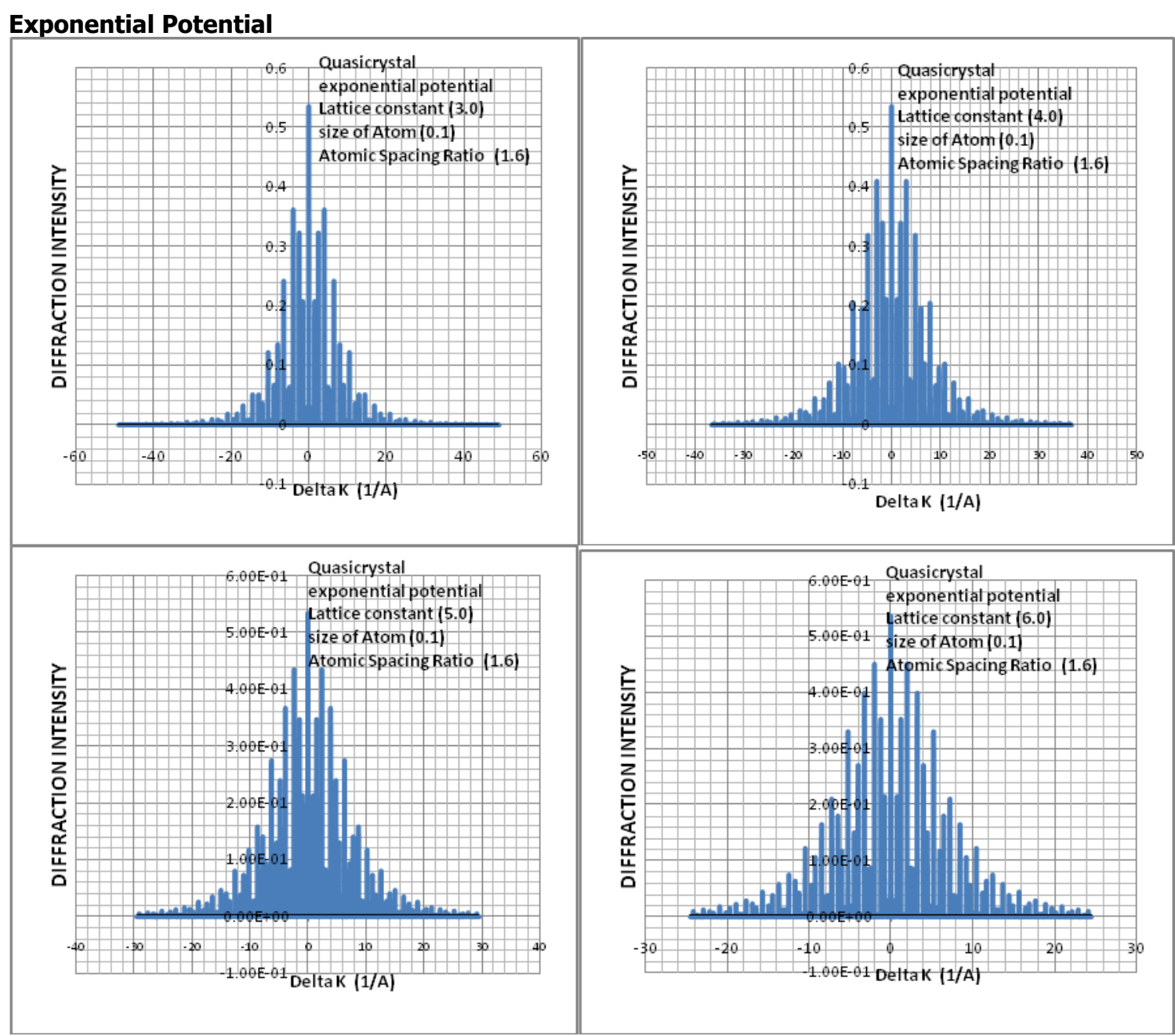

Figure 4: Sample of the Diffraction Patterns for Exponential Potential

The following features of the diffraction pattern of the Quasicrystal (atomic potential being Pseudo) are observed:

i. Each diffraction pattern has sharp peaks, just like periodic crystal, but in addition there is a background intensity.

ii. The spatial distribution of the diffraction peaks is unique for a given size of atom, in particular the spacing between the peaks is constant for a given size of atom and independent of the lattice constant

iii. Intensity of the central peak was found to be constant and the same as that of Gaussian and Pseudo potentials.

iv. For a given size of the atom, there is intermediate (greater than Gaussian but less than Pseudo) increase in the background intensity, as the lattice constant increases. 
v. For a given lattice constant, the width of the diffraction pattern decreases (however very slow) with increase in size of atom. However, for a given size of atom the width decreases sharply on increasing the lattice constant as shown in Fig. 4..

Table 4: Variation of width of diffraction patter with size of the atom

\begin{tabular}{|c|c|c|c|c|c|}
\hline $\begin{array}{c}\text { Lattice constant } \\
(A)\end{array}$ & $\mathbf{3 . 0}$ & $\mathbf{4 . 0}$ & $\mathbf{5 . 0}$ & $\mathbf{6 . 0}$ & $\mathbf{7 . 0}$ \\
\hline & & & & & \\
\hline & & & & & \\
\hline 0.1 & & & & & \\
\hline 0.2 & 98.1059 & 73.5794 & 58.8779 & 49.0605 & 42.0557 \\
\hline 0.3 & 96.9078 & 73.2918 & 58.6334 & 49.0530 & 42.0454 \\
\hline 0.4 & 96.7160 & 72.6808 & 58.6334 & 48.8612 & 41.8810 \\
\hline 0.5 & 96.7160 & 72.5370 & 58.0296 & 48.3580 & 41.5318 \\
\hline 0.6 & 96.7160 & 72.5370 & 58.0296 & 48.3580 & 41.5318 \\
\hline 0.7 & 96.7160 & 72.5370 & 58.0296 & 48.3580 & 41.4498 \\
\hline & 91.7796 & 72.5370 & 58.0296 & 48.3580 & 41.4498 \\
\hline
\end{tabular}

\section{DISCUSSION}

The following major differences between the diffraction patterns of crystal and a quasicrystal have been observed in this work:

(i) In the periodic monatomic chain, the diffraction pattern consists of descrete peaks separated by regions of zero intensity, while in quasiperiodic monatomic chain, there is in addition to these peaks, a background intensity so that in the region bounded by the width of the diffraction pattern, the intensity is non zero everywhere. The quasicrystal reciprocal lattice is very dense to the extent that the wave vectors form a continuous spectrum, therefore, the allowed scattering wave vectors are also continuous.

(ii) In quasicrystals, the intensity of the central peak depends on the golden mean i.e the spacing ratio is constant for a given golden mean (Sa'id, 2012). In this work, the intensity of the central peak for the quasiperiodic chains was found to be a constant of 0.54 since the golden mean is set at 1.6 only. However in the periodic monatomic chain, the intensity of the central peak was found to be not constant (depends on both the size of the atom and the lattice constant). However in the plots of the diffraction patterns shown in Fig.1.0, the intensity of the central peak has been normalized to 1.0 .

(iii) In both chains (periodic and quasi), the intensity of the central peak is greater than that of the other peaks. Let $\left|\Delta k_{1}\right|<\left|\Delta k_{2}\right|<\left|\Delta \mathrm{k}_{3}\right|$. In case of one-dimensional periodic chain, the intensity of the peaks are always such that $I_{\Delta k_{11}}>I_{\Delta k_{2}}>I_{\Delta k_{n}}$, however, in the quasichain it is not necessarily. In fact, it is possible for $I_{\Delta k_{g}}>I_{\Delta k_{1}}$.

(iv) The width and the modulated shape of the diffraction pattern formed by a quasicrystal depends on the atomic potential. For guassian and exponential atomic shapes, the width decreases as the lattice constant is increased. It also decreases as the size of the atom is increased. For pseudo-atomic shape, the width vary with lattice constant and size of the atom in an un-orderly manner.The maximum width in Figure( $1-4)$ is found when the lattice constant is $3.0 . A$ and the atomic size is $0.1 A$. The maximum width in Fig. 4, and Table 4.is 98.1059A was produced by Exponential Potentials. The Guassian produced a maximum width in Fig. 2 and Table 2 . is $70.835 \mathrm{~A}$. The modulated shape of the diffraction pattern is found to be the same as the shape of the atomic potential distribution. For example the Guassian Potential produces a diffraction pattern whose modulated shape is the same as shape of the Gaussian distribution.

As stated above, the shape of the atomic potential affects the width and the modulated shape of the diffraction pattern of the one-dimensional quasicrystal. The manner in which the shape of the atomic potential affects these two features is now discussed.

\section{- Gaussian Potential}

For a small value of lattice constant in Fig.2 and Table. 2, the width of the diffraction drops very fast as the size of the atom increases. Also at a very large size of the atom, the diffraction width increases as the lattice constant increases. This is because the atomic size is larger compared to the X-ray wave length, waves scattered from different part of the atom will interfere destructively. In this case the scattered amplitude from single atom is inversely proportional to the number of electrons and hence the atomic number Z.

\section{- Pseudo Potential}

The diffraction width drops very slowly as the size of the atom increases, however it drops very fast as the lattice constant increases. Also when compared with Gaussian potential it was found that the diffraction width is four times larger. This is because the pseudo potential gives information not only on the surface of the atom but also the nucleon. 


\section{- Exponential Potential}

The diffraction width of exponential potential is comparable to that of pseudo potential but differs only in the intensity.

\section{CONCLUSION}

In this work, the diffraction patterns of periodic and quasiperiodic monatomic chains of atoms were simulated and the effects of the shape of the atomic potential investigated. The following are our conclusions:

- The code Leue, written by Silsbee and Drager (2005) has been found to be not only suitable but also accurate in simulating the diffraction pattern of one-

\section{REFERENCES}

De-Bruijn, N.G., Ned., Acad.(1981),Weten. Proc. Ser. A43,39,53.

Diehl, R. D., Ledieu, J., Ferralis. N.,Szmodis. A.W, and Mc Garth. R.(2003), Low-energy-electron diffraction from Quasicrystal surface, J. phys. Condens matter 15 (3) R63-R81.

Dueau, M. and Katz A.(1984), Phys. Rev. Lett. 54, 2688.

Ferralis, N., Szmodis, A., W and Diehl. R. D.,(2004),Diffraction from one- and twodimensional quasicrystalline gratings,American Association of Physics Teachers, University Pennsylvania, 16802, 1241-1246.

Fujiwara, T. and Yasushi, I.(2008), Introduction to Quasicrystal. Handbook of Metal Physics. Elservier, Iss1570-002X/DOI 10.1016/S1570002X(06) 0200-5.

Ishimasa, T., Nissen, H.U.andFukano,Y.(1985), New ordered state between Crystalline and amorphous in $\mathrm{Ni}$-Cr particles, Physical Review Letter 55(5): 511-513.

Kramer, P. andNeri, R.(1984), ActaCrystallogr. A40, 580.

Latvin, S.Y.(1988), Generation and Experimental Measurement of One-Dimensional Quasicrystal Diffraction Pattern, Am. J. phys.56(1), 72-75.

Lifshitz, R. and Even-Dar Mandel.S.(2004),Symmetry of magnetically ordered quasicrystals, ActaCryst.A60, 167.

Lifshitz, R.(2003), Quasicrystal; A matter of definition, Found.Phys.33 (12) 1703-1711. dimensional monatomic chain of atoms when compared with theoretical approaches.

- The width and the modulated shape of the diffraction pattern of quasiperiodic monatomic chain of atoms depends on the shape of the atomic potential.

- In general, the effects of the Pseudo atomic potential are " intermediate " compared to the effects of the guassian and exponential potentials.

- The width of the diffraction pattern of quasiperiodic chain with an exponential or Gaussian atomic shape varies in an orderly manner with respect to lattice constant and size of the atom. However, the width of the pattern for a chain with Pseudo atomic potential varies in an un-orderly manner with respect to lattice constant and size of the atom.

Livine, D.(1985), Quasicrystals, Journal De Physique, vol. 46 no. 8, 397-402.

Mermin, N.D., Rev. (1992).Mod. Phys. 64,1051.

Said, M. (2012). Simulation of the Diffraction Pattern of one-dimensional Quasicrystal Material, Published M.Sc Dissertation, Bayero University, Kano. Chapter four.

Shechtman, D., Blech, I., Gratias, D., and Cahn, J.(1984), Rev. let. 531951.

Silsbee, R. H. and Drager,J.(2005), Simulations for Solid State Physics, Cambridge University Press, (London). Chapterthree.

www.lassp.cornell.edu/Lifshtz/quasicrystal.html-6 retrive12/02/2012 